\title{
Synchronous Bilateral Breast Carcinoma and Axillary Non-Hodgkin Lymphoma: A Case Report and Review of the Literature
}

\author{
Edward F. Miles ${ }^{1}$ and Laura L. Jacimore ${ }^{2}$ \\ ${ }^{1}$ Division of Radiation Oncology, Department of Radiology, Naval Medical Center Portsmouth, 620 John Paul Jones Circle, Portsmouth, \\ VA 23708, USA \\ ${ }^{2}$ Department of Radiation Oncology, Nash General Hospital, 2460 Curtis Ellis Drive, Rocky Mount, NC 27804, USA
}

Correspondence should be addressed to Edward F. Miles, edward.miles@med.navy.mil

Received 26 June 2012; Accepted 29 August 2012

Academic Editors: J. M. Buchanich, E. Itakura, D. V. Jones, and G. P. Vandoros

Copyright ( 2012 E. F. Miles and L. L. Jacimore. This is an open access article distributed under the Creative Commons Attribution License, which permits unrestricted use, distribution, and reproduction in any medium, provided the original work is properly cited.

\begin{abstract}
The use of staging imaging modalities with increased sensitivity has led to an increase in the incidence of detection of simultaneous malignancies. These cases require careful evaluation and discussion in a multidisciplinary setting to establish a treatment plan that optimizes the outcome with respect to each malignancy, particularly when treatment modalities overlap. We report a case of a patient diagnosed with axillary nodal diffuse large B-cell lymphoma (DLBCL) in a community hospital where staging workup also revealed synchronous bilateral breast carcinomas. To our knowledge, this is only the second case report of a patient with three synchronous primary malignancies: bilateral breast carcinomas and axillary DLBCL. The only other similar case report had no role for radiation or chemotherapy in the management of the indolent follicular lymphoma.
\end{abstract}

\section{Introduction}

Breast cancer constitutes approximately $14 \%$ of all primary cancers in women, accounting for more than 207,000 cases per year [1], but only a small fraction $(2.2-4.3 \%$ in retrospective reviews) of these presented as bilateral breast cancer [2-4]. For early-stage disease, primary treatment involves surgical resection via modified radical mastectomy or breast conservation therapy with lumpectomy and sentinel lymph node biopsy. Adjuvant chemotherapy and hormonal therapy are often considered as well as completion of breast conservation therapy with radiation therapy to the involved breast and, if indicated, the regional lymph nodes [5]. Conversely, non-Hodgkin lymphoma constitutes only $4 \%$ of all primary cancers in women. Primary treatment for limited stage diffuse large B-cell lymphoma (DLBCL) involves systemic chemotherapy with or without consolidative radiation therapy, while late stage is treated primarily with systemic chemotherapy alone with radiation therapy considered for areas of original bulky disease [6].
Herein, we report a case of a patient diagnosed with axillary nodal DLBCL in a community hospital where staging workup also revealed synchronous bilateral breast carcinomas. A review of the relevant literature is also discussed.

\section{Case Report}

The patient was a 64-year-old woman who presented to her local community hospital emergency department with a complaint of rapidly increasing edema to the right arm. She was noted to have significant right axillary adenopathy and although she denied fevers or night sweats, she had lost over 25 pounds ( $>10 \%$ of her body weight) in the three months prior to presentation. A computed tomography (CT) scan of the chest demonstrated multiple right axillary nodes and a questionable mass in the left breast. She underwent excisional biopsy of a right axillary node which demonstrated DLBCL (Figure 1). Her bone marrow biopsy was negative. Due to the findings on her staging CT scan and 


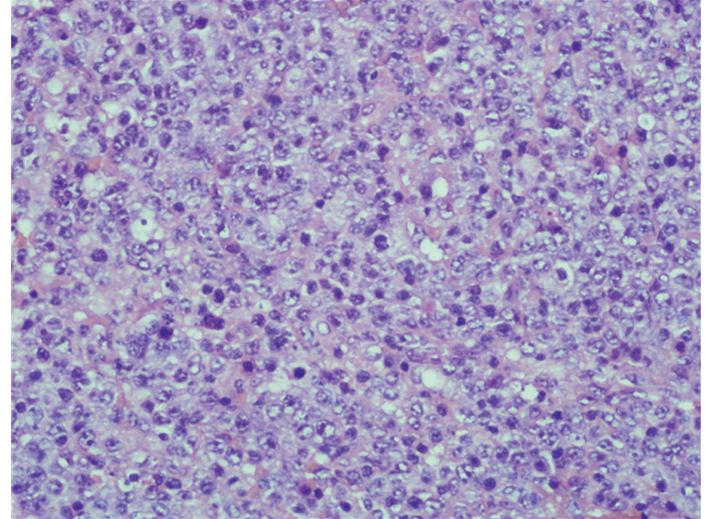

FIGURE 1: Right axillary node biopsy demonstrating diffuse large Bcell lymphoma.

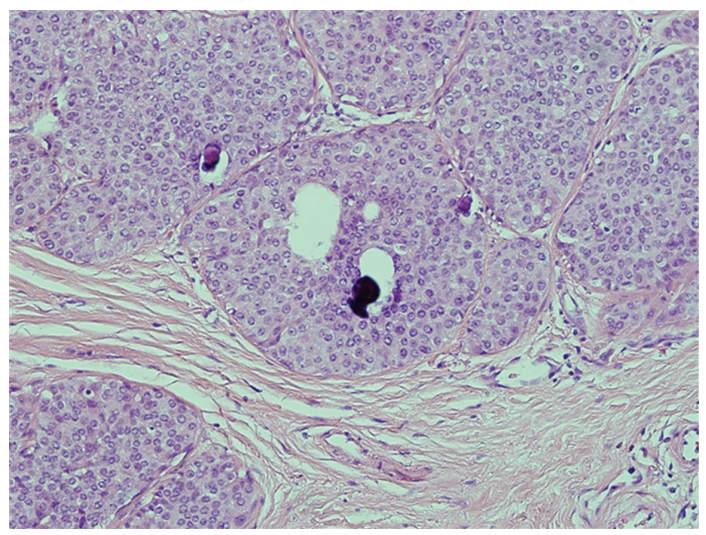

FIgURE 2: Right breast ductal carcinoma in situ.

a palpable mass in the left breast, a bilateral mammogram was performed that showed a solid density in the vicinity of the left breast mass, but also showed calcifications in the medial right breast. Ultrasound-guided needle core biopsy of the breast lesions demonstrated ductal carcinoma in situ (DCIS) in the right breast (Figure 2), and estrogen and progesterone receptor positive, HER2 receptor negative infiltrating ductal carcinoma in the left breast (Figure 3). Due to issues with insurance and the relatively urgent need to treat her presumably Stage IB lymphoma, systemic chemotherapy with R-CHOP (rituximab, cyclophosphamide, adriamycin, vincristine, and prednisone) was started prior to a staging positron emission tomography (PET)/CT scan. Subsequent PET/CT scan showed a questionable area of "thickening" in the retrocrural area worrisome for lymphomatous involvement. She had a dramatic improvement in the right arm edema after the first cycle of chemotherapy. She continued systemic therapy for three cycles and then underwent a lumpectomy for the DCIS in the right breast (stage 0) and simple mastectomy with $2.1 \mathrm{~cm}$ of invasive disease with a negative sentinel lymph node biopsy on the left (stage IIa). Unfortunately, pathology revealed that the anterior margins for both resections were very close at less than 1 millimeter. Her case was discussed at a multidisciplinary

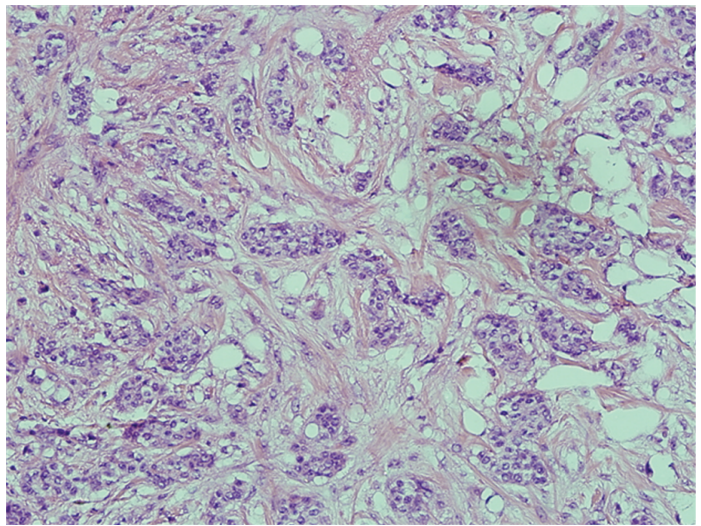

Figure 3: Left breast infiltrating ductal carcinoma.

tumor board, and a recommendation was made to reresect for clear margins bilaterally, and then due to the unfavorable risk factors for her DLBCL (age greater than 60 and B symptoms) and possible stage IIIB at diagnosis, to continue her systemic chemotherapy for an additional three cycles. PET/CT scan after completion of therapy revealed resolution of the retrocural thickening, indicating possible stage IIIB disease at diagnosis and two remaining PET-avid nodes in the right axilla. She underwent limited axillary dissection to determine her disease status and only necrotic tissue was found with no evidence of malignancy. Completion of her breast conservation therapy with standard breast tangent fields [7] allowed for incidental inclusion of levels I and II of the axilla in the treatment field with $88 \%$ of the right axilla receiving 3,000 cGy or more (Figure 4). With a mastectomy and negative sentinel lymph node biopsy for the left breast, local control therapy for this malignancy was considered complete. On the right, she had undergone breast conservation therapy, and her local therapy was completed with radiation therapy to the right breast. Routine followup has shown no clinical or radiographic evidence of any of her malignancies, and she continues on hormonal therapy for her receptor positive infiltrating ductal carcinoma.

\section{Discussion}

As the use of PET/CT scans for the staging of malignancies has become more common, the detection of second occult malignancies has also increased. A recent prospective study of non-Hodgkin lymphoma patients staged by PET/CT demonstrated that $2.9 \%$ of them had a second, occult, nonlymphoma malignancy [8]. Although there are wellestablished guidelines for the workup, staging, and treatment of individual malignancies, optimal treatment in the setting of multiple simultaneous malignancies is difficult. There are often competing urgency requirements, and the most pressing issue is treated first with an eye towards optimizing treatment for each malignancy as much as is possible. In this case, the rapidly expanding right arm edema secondary to her axillary lymphadenopathy from her DLBCL was this patients' most pressing issue. Per the National 


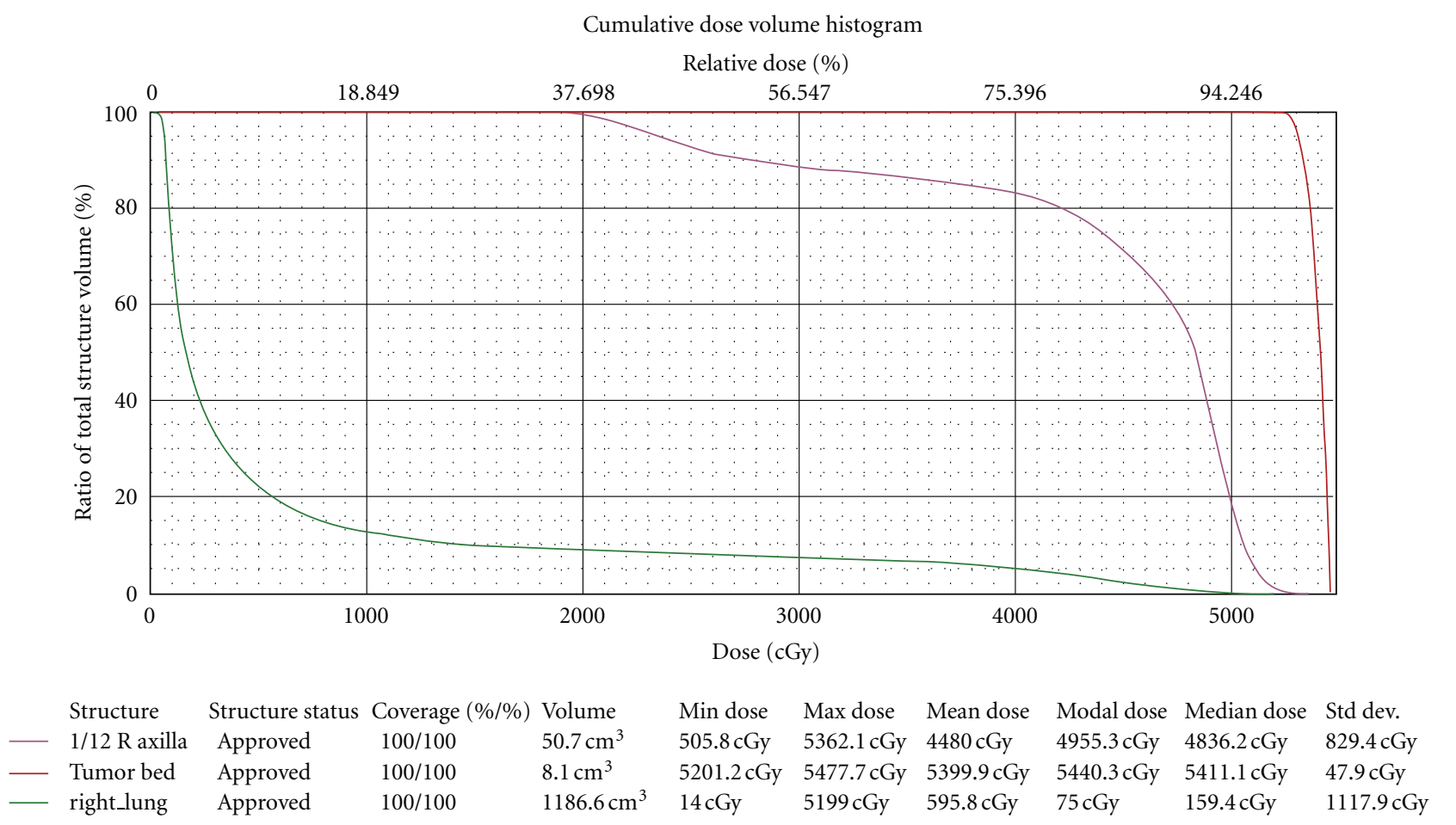

FIGURE 4: Dose volume histogram showing coverage of breast tumor bed (red) with standard breast tangents and incidental ipsilateral axillary coverage (purple).

Comprehensive Cancer Network (NCCN) clinical practice guidelines, the standard of care for first-line treatment of Stage IB DLBCL in a patient with two adverse risk factors is systemic chemotherapy with R-CHOP for three cycles with involved field radiation therapy or six cycles with or without radiation therapy; if her retrocrural node was assumed to be positive, this would give her stage IIIB disease, and systemic chemotherapy for 6-8 cycles would have been indicated, with radiation considered to areas of initially bulky disease [6]. The systemic chemotherapy which was administered for treatment of the DLBCL also doubled as neoadjuvant and adjuvant therapy for the invasive ductal carcinoma, as three of the agents delivered (cyclophosphamide, doxorubicin, and vincristine) are active in breast cancer, and cyclophosphamide and doxorubicin are a standard regimen for resectable breast cancer. There is no role for systemic chemotherapy in DCIS.

The literature contains several small case series and individual case reports of patients with primary breast malignancies with synchronous lymphoproliferative disorders including follicular lymphoma [9-11], small lymphocytic lymphoma/chronic lymphocytic leukemia [10, 12], Hodgkin lymphoma [10], mantle cell lymphoma [11], marginal zone lymphoma [13], and mucosa-associated lymphoid tissue (MALT) lymphoma [14, 15]. Two individual case reports document primary breast carcinoma with simultaneous DLBCL; these both occurred in the breast rather than in the axilla $[16,17]$. The single case report that does discuss therapy indicates that six cycles of $\mathrm{CHOP}$ chemotherapy were administered as well as tamoxifen for hormone receptor positive invasive breast cancer. To our knowledge, this is only the second case report of a patient with three synchronous primary malignancies: bilateral breast carcinomas and axillary DLBCL. The only other case report had no role for radiation or chemotherapy in the management of the more indolent (follicular) lymphoma [13].

In conclusion, the appropriate use of sensitive staging studies makes the discovery of occult simultaneous malignancies a distinct possibility. Careful review of these studies with evaluation and discussion in a multidisciplinary setting ensures the most efficacious treatment regimen is planned and executed to maximize the chance of cure of these malignancies.

\section{Conflict of Interests}

The views expressed in this paper are those of the author(s) and do not necessarily reflect the official policy or position of the Department of the Navy, Department of Defense, or the United States Government. E. F. Miles is a military service member. This work was prepared as part of his official duties. Title 17 U.S.C. 105 provides that "Copyright protection under this title is not available for any work of the United States Government." Title 17 U.S.C. 101 defines a United States Government work as a work prepared by a military service member or employee of the United States Government as part of that person's official duties. Work performed at the Naval Medical Center Portsmouth, Portsmouth, VA and Nash General Hospital, Rocky Mount, NC. 


\section{References}

[1] A. Jemal, R. Siegel, E. Ward, Y. Hao, J. Xu, and M. J. Thun, "Cancer statistics, 2009," CA: Cancer Journal for Clinicians, vol. 59, no. 4, pp. 225-249, 2009.

[2] L. Schwentner, R. Wolters, M. Wischnewsky et al., "Survival of patients with bilateral versus unilateral breast cancer and impact of guideline adherent adjuvant treatment: a multicentre cohort study of 5292 patients," Breast, vol. 21, no. 2, pp. 171-177, 2012.

[3] W. Setz-Pels, L. E. M. Duijm, J. H. Groenewoud et al., "Patient and tumor characteristics of bilateral breast cancer at screening mammography in the Netherlands, a populationbased study," Breast Cancer Research and Treatment, vol. 129, no. 3, pp. 955-961, 2011.

[4] R. Díaz, B. Munárriz, A. Santaballa, L. Palomar, and J. Montalar, "Synchronous and metachronous bilateral breast cancer: a long-term single-institution experience," Medical Oncology, vol. 29, no. 1, pp. 16-24, 2010.

[5] R. W. Carlson, D. C. Allred, B. O. Anderson et al., "Breast cancer: clinical practice guidelines in oncology," Journal of the National Comprehensive Cancer Network, vol. 7, pp. 122-192, 2009.

[6] A. D. Zelenetz, J. S. Abramson, R. H. Advani et al., "NCCN clinical practice guidelines in oncology: non-Hodgkin's lymphomas," Journal of the National Comprehensive Cancer Network, vol. 8, pp. 288-334, 2010.

[7] B. Fisher, J. Dignam, N. Wolmark et al., "Lumpectomy and radiation therapy for the treatment of intraductal breast cancer: findings from National Surgical Adjuvant Breast and Bowel Project B- 17," Journal of Clinical Oncology, vol. 16, no. 2, pp. 441-452, 1998.

[8] T. Papajík, M. Mysliveček, Z. Sšedová et al., "Synchronous second primary neoplasms detected by initial staging F-18 FDG PET/CT examination in patients with non-Hodgkin lymphoma," Clinical Nuclear Medicine, vol. 36, no. 7, pp. 509$512,2011$.

[9] M. D. Laudenschlager, K. L. Tyler, M. C. Geis, M. R. Koch, and D. B. Graham, "A rare case of synchronous invasive ductal carcinoma of the breast and follicular lymphoma," South Dakota Medicine, vol. 63, no. 4, pp. 123-125, 2010.

[10] K. E. Cuff, A. J. Dettrick, and B. Chern, "Synchronous breast cancer and lymphoma: a case series and a review of the literature," Journal of Clinical Pathology, vol. 63, no. 6, pp. 555$557,2010$.

[11] J. Cox, L. Lunt, and L. Webb, "Synchronous presentation of breast carcinoma and lymphoma in the axillary nodes," Breast, vol. 15, no. 2, pp. 246-252, 2006.

[12] D. Wahner-Roedler, C. Reynolds, and J. Boughey, "Collision tumors with synchronous presentation of breast carcinoma and lymphoproliferative disorders in the axillary nodes of patients with newly diagnosed breast cancer: a case series," Clinical Breast Cancer, vol. 11, no. 1, pp. 61-66, 2011.

[13] N. K. Garg, N. B. Bagul, G. Rubin, and E. F. Shah, "Primary lymphoma of the breast involving both axillae with bilateral breast carcinoma," World Journal of Surgical Oncology, vol. 6, article 52, 2008.

[14] B. Susnik, J. J. Rowe, P. N. Redlich, C. Chitambar, C. C. Chang, and B. Kampalath, "A unique collision tumor in breast: invasive ductal carcinoma and mucosa-associated lymphoid tissue lymphoma," Archives of Pathology and Laboratory Medicine, vol. 128, no. 1, pp. 99-101, 2004.

[15] J. M. Quilon, T. A. Gaskin, A. S. Ludwig, and C. Alley, "Collision tumor: invasive ductal carcinoma in association with mucosa-associated lymphoid tissue (MALT) lymphoma in the same breast," Southern Medical Journal, vol. 99, no. 2, pp. 164-167, 2006.

[16] F. A. Siddiqui, V. Maheshwari, K. Alam et al., "Coexistent nonHodgkins lymphoma and ductal carcinoma breast: diagnosis on fine needle aspiration cytology," Diagnostic Cytopathology, vol. 39, no. 10, pp. 767-769, 2011.

[17] S. Broco, N. Bonito, P. Jacinto, G. Sousa, and H. Gervásio, "Primary non-Hodgkin lymphoma and invasive ductal carcinoma in the same breast: a rare case report," Clinical and Translational Oncology, vol. 11, no. 3, pp. 186-188, 2009. 


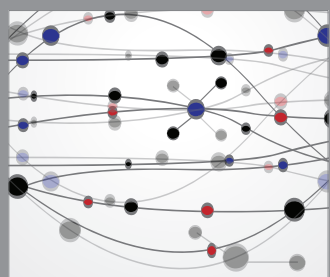

The Scientific World Journal
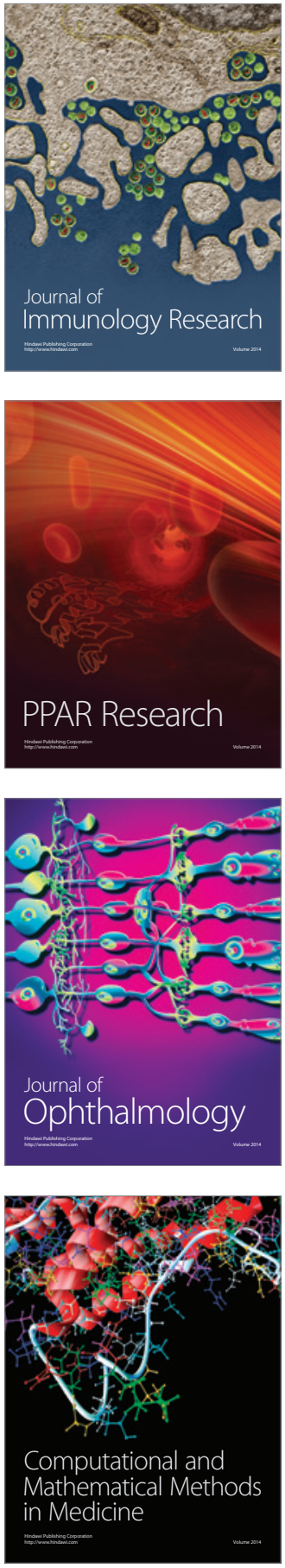

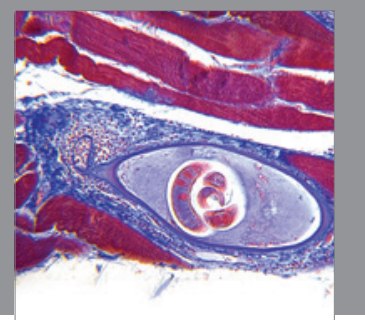

Gastroenterology

Research and Practice
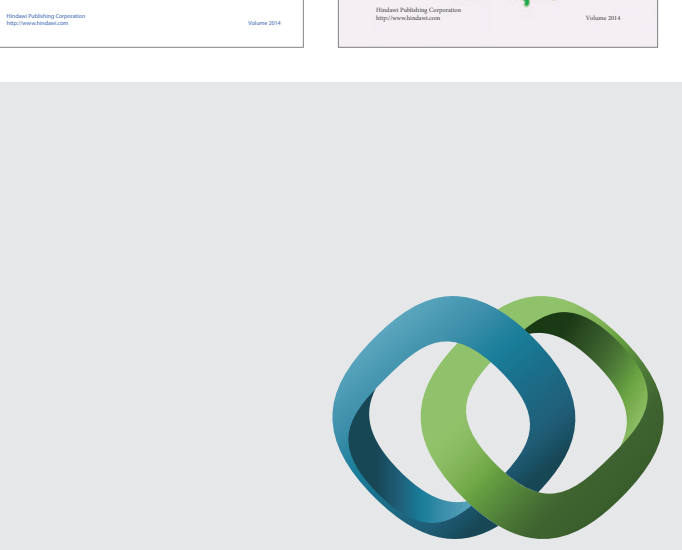

\section{Hindawi}

Submit your manuscripts at

http://www.hindawi.com
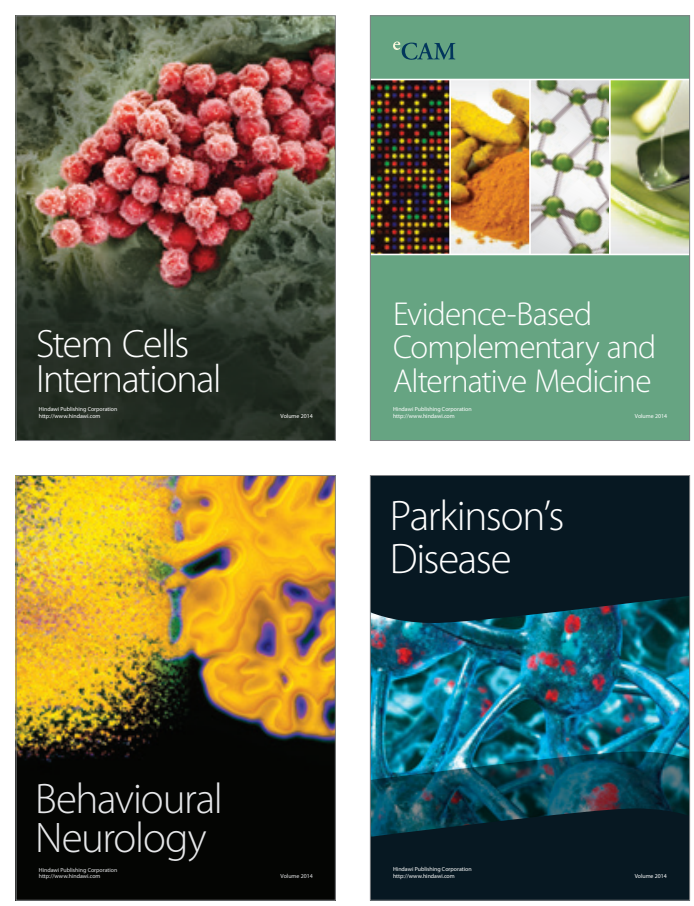

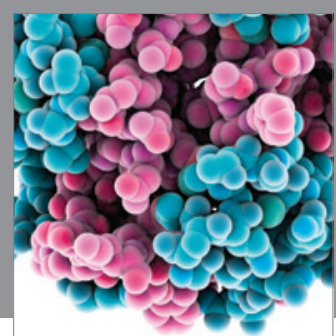

Journal of
Diabetes Research

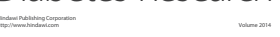

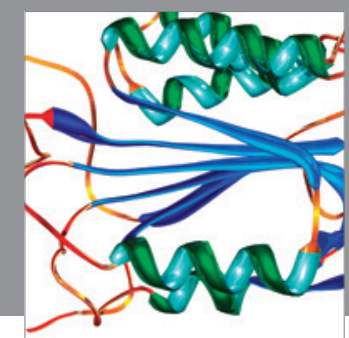

Disease Markers
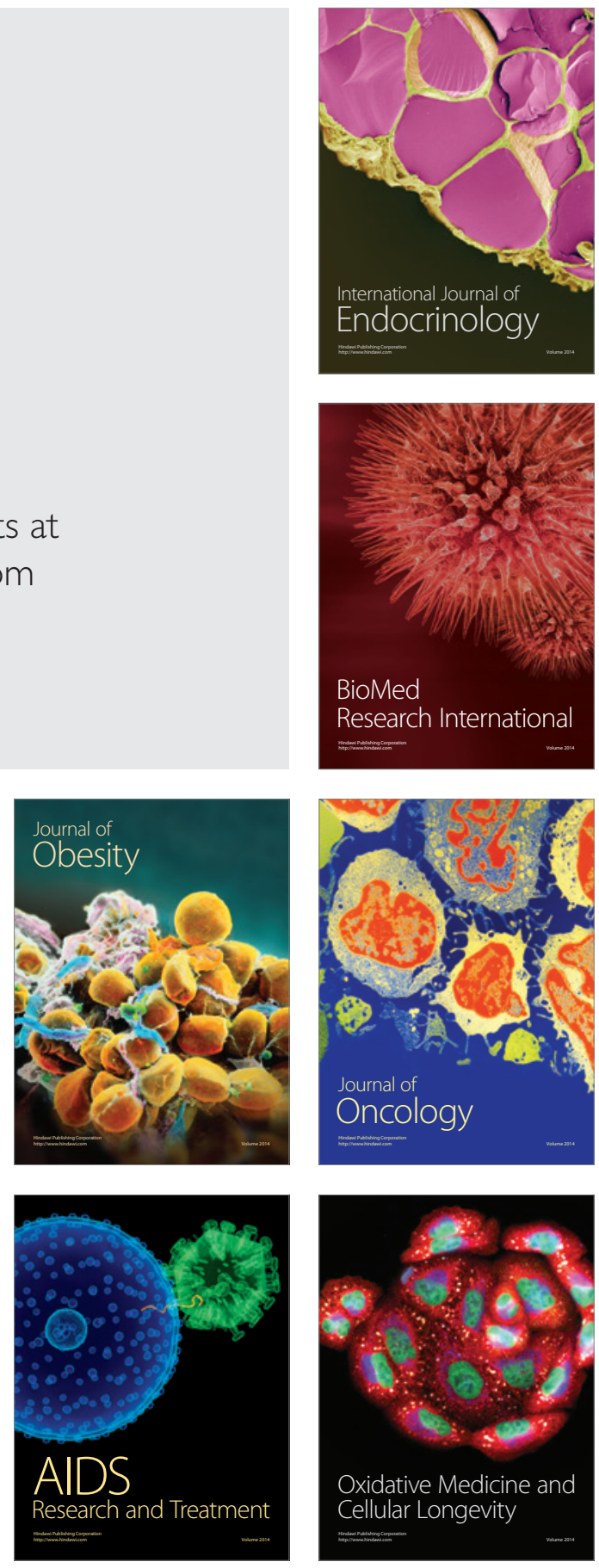